\title{
Increased Conformational Rigidity of Humic Substances by Oxidative Biomimetic Catalysis
}

\author{
Alessandro Piccolo, ${ }^{*}, \dagger$ Pellegrino Conte,${ }^{\dagger}$ and Pietro Tagliatesta ${ }^{\ddagger}$ \\ Dipartimento di Scienze del Suolo, della Pianta e dell'Ambiente, Università di Napoli Federico II, \\ Via Università 100, 80055 Portici, Italy, and Dipartimento di Scienze e Tecnologie Chimiche, \\ Università di Roma Tor Vergata, Via della Ricerca Scientifica, 00187 Roma, Italy
}

Received August 17, 2004; Revised Manuscript Received October 7, 2004

\begin{abstract}
A synthetic water-soluble meso-tetra(2,6-dichloro-3-sulfonatophenyl)porphyrinate of iron(III) chloride, Fe(TDCPPS)Cl, was employed as a biomimetic catalyst in the oxidative coupling of terrestrial humic materials. High-performance size-exclusion chromatography (HPSEC), solid-state nuclear magnetic resonance (CPMAS${ }^{13} \mathrm{C}$ NMR), electron paramagnetic resonance (EPR), and diffuse reflectance infrared spectroscopy (DRIFT) were used to follow conformational and structural changes brought about in different humic materials by the oxidative coupling. Increase in apparent weight-average molecular weight $\left(\mathrm{Mw}_{\mathrm{a}}\right)$ occurred invariably for all humic substances with the oxidative polymerization catalyzed by Fe(TDCPPS)Cl. HPSEC further showed that the polymerization reaction turned the loosely bound humic supramolecular structures into more stable conformations which could no longer be disrupted by the disaggregating effect of acetic acid. DRIFT spectroscopy suggested the formation of new alkyl and aromatic ethers following the oxidative coupling with the biomimetic catalyst. CPMAS- ${ }^{13} \mathrm{C}$ NMR and EPR spectra suggested a reduced molecular mobility of humic components and enhanced stabilization of free radicals in larger oxidized fragments. All findings concur in indicating that the biomimetic catalysis by $\mathrm{Fe}(\mathrm{TDCPPS}) \mathrm{Cl}$ increased the molecular mass and chemical rigidity of humic materials by formation of intermolecular covalent bonds via a free-radical mechanism. The development of a technology based on oxidative polymerization by biomimetic catalysis may be of importance in controlling the properties and reactivity of humic matter for industrial and environmental applications.
\end{abstract}

\section{Introduction}

Humic substances (HS) are naturally occurring supramolecular structures, ubiquitous in water, soil, and sediments, of paramount importance in controlling both the fate of environmental pollutants and the biogeochemistry of organic carbon in the global ecosystem. Humic substances are increasingly studied for applications in sustainable technologies. ${ }^{1,2}$

It has been recently proposed that HS rather than being constituted by macromolecular polymers, as traditionally believed, may be better described as supramolecular associations of relatively small heterogeneous molecules. ${ }^{3-6}$ The supramolecular nature implies that humic molecules, instead of being covalently interlinked, are randomly associated by weak dispersive forces (van der Waals, $\pi-\pi, \mathrm{CH} / \pi$ ) in only apparent high molecular dimensions which can be reversibly disrupted by interacting with natural organic acids. ${ }^{7,8}$ Disruption of apparently large dimensions of humic material into smaller components was also achieved by extracting divalent metals with strong chelating agents. ${ }^{9}$ The action of organic acids on the humic conformational structure not only

\footnotetext{
* Corresponding author. Telephone: +39-081-2539160. E-mail alpiccol@unina.it.

† Università di Napoli Federico II.

†niversità di Roma Tor Vergata.
}

significantly affects the fluorescence and thermal behavior of $\mathrm{HS}^{10,11}$ but also provides a chemical method to fractionate the bulk of a humic material in less heterogeneous size fractions of different composition ${ }^{12}$ and average molecular masses between 700 and $1200 \mathrm{Da}^{13}$

The supramolecular understanding of HS suggests the possibility of increasing the size of humic molecules by polymerization reactions. It is known that a number of naturally occurring phenolic monomers can undergo oxidative coupling reactions catalyzed by oxidative enzymes such as laccases and peroxidases to produce polyphenols consisting of phenylene and oxyphenylene units. ${ }^{14-17}$ Laccases and peroxidases are thought to couple phenolic compounds by way of a radical mechanism. ${ }^{18}$ Thus, it may be expected that phenolic compounds present in the loosely bound humic supramolecular structures would undergo the same radicalmediated intermolecular polymerization observed by phenolic monomers when catalyzed by the enzymes. An increase in molecular size of humic matter following an oxidative polymerization reaction catalyzed by peroxidase was effectively observed by employing size-exclusion chromatography and infrared spectroscopy. ${ }^{19,20}$ Contrary to the original humic material, interaction with an organic acid had little effect on the size distribution of the polymerized humic matter, thereby indicating that its conformation should be stabilized by newly formed covalent bonds. 
It may be more efficient and economical to mimic oxidase enzymes by employing synthetic water-soluble biomimetic catalysts which contain an active metal-porphyrin ring. Iron-porphyrin, a nontoxic compound, has been already applied to dechlorinate and to couple chlorophenols ${ }^{21-23}$ and other organic compounds. ${ }^{21,24,25}$ It is believed that the mechanism of action of iron-porphyrins is induced by an oxygen donor producing high-valent iron-oxo species which become the active oxidant of phenolic moieties. ${ }^{26}$ As for the case of phenoloxidase enzymes, the oxidized phenols can undergo further couplings via a radical mechanism. ${ }^{27}$

Our aim was to subject different HS to an oxidative coupling reaction catalyzed by a biomimetic water-soluble iron-porphyrin and to verify the occurrence of macromolecules in the complex supramolecular structure of humic materials by chromatographic and spectroscopic techniques.

\section{Experimental Section}

Humic Substances. Three humic acids (HAs) were isolated from different raw materials: HA1 from an oxidized coal provided by Eniricerche SpA (Italy), HA2 from North Dakota Leonardite (Mammoth, International Chemical Co.), and HA3 from a volcanic soil (Typic Xerofluvent) near Rome (Italy). The HAs were extracted and purified by common procedures as described elsewhere. ${ }^{3}$ The HAs were redissolved in $0.5 \mathrm{M} \mathrm{NaOH}$ under $\mathrm{N}_{2}$ and passed through a strong cation-exchange resin (Dowex 50) to further eliminate divalent and trivalent metals and freeze-dried again. Elemental characterizations by elemental analyzer and carbon molecular distribution by CPMAS $-{ }^{13} \mathrm{C}$ NMR spectrometry were previously reported. ${ }^{20}$ Purified HA samples $(50 \mathrm{mg}$ ) were subsequently suspended in distilled water $(50 \mathrm{~mL})$ and titrated to $\mathrm{pH} 7$ by an automatic titrator (VIT 90 Videotitrator, Radiometer, Copenhagen, Denmark), with a $\mathrm{CO}_{2}$-free 0.5 $\mathrm{M} \mathrm{NaOH}$ solution under $\mathrm{N}_{2}$ atmosphere and continuous stirring. After having reached the constant $\mathrm{pH} 7$, the solution containing sodium humates was left under titration for $2 \mathrm{~h}$, filtered through a Millipore $0.45 \mu \mathrm{m}$ filter, and freeze-dried.

Water-Soluble Iron(III)-Porphyrin, Fe(TDCPPS)Cl. meso-Tetra(2,6-dichlorophenyl)porphyrin ( $\left.\mathrm{H}_{2} \mathrm{TDCPP}\right)$ was synthesized according to the methods reported by Traylor et al. ${ }^{24}$ The $\mathrm{H}_{2}$ TDCPP (200 mg) was then dissolved in $8 \mathrm{~mL}$ of steaming $\mathrm{H}_{2} \mathrm{SO}_{4}$ and stirred for $12 \mathrm{~h}$ at $160{ }^{\circ} \mathrm{C}$ under argon atmosphere. The product was recovered with cool water in a flask immersed in ice. The solution $\mathrm{pH}$ was increased up to 7 with a saturated $\mathrm{NaOH}$ solution and then evaporated at $60-70{ }^{\circ} \mathrm{C}$ under vacuum. The residue was recovered with methanol, again evaporated under vacuum, re-dissolved in methanol, and recrystallized in ethyl ether. The product was obtained by filtration and then purified through a cationic exchange resin Dowex 50W X8-100 (50-100 mesh), previously conditioned with a $10 \% \mathrm{HCl}$ solution. The product was eluted with water and the recovered fraction dried under vacuum. The final material, meso-tetra(2,6-dichloro-3-sulfonatophenyl)porphyrin ( $\mathrm{H}_{2}$ TDCPPS), was recrystallized in a methanol-acetone solution with a final reaction yield of $75 \%$.

The $\mathrm{H}_{2}$ TDCPPS (200 mg) and $100 \mathrm{mg}$ of $\mathrm{Fe}^{\mathrm{II}} \mathrm{SO}_{4}$ were dissolved in $100 \mathrm{~mL}$ of water. The solution was degassed and left $12 \mathrm{~h}$ under argon atmosphere. Then the solution was vacuum-evaporated and the residue re-dissolved in water. This solution was filtered and purified through a cationic exchange resin Dowex 50W X8-100 (50-100 mesh), previously conditioned with a $10 \% \mathrm{HCl}$ solution. The column was eluted with water and the recovered material dried upon vacuum. The final product meso-tetra(2,6-dichloro-3-sulfonatophenyl)porphyrinate of iron(III) chloride, Fe(TDCPP$\mathrm{S}) \mathrm{Cl}$, was recrystallized in methanol-acetone in order to purify the sample from residual salts. The yield of the reaction was $80 \%$. The purity of $0.15 \mathrm{mg} \cdot \mathrm{mL}^{-1} \mathrm{Fe}-$ (TDCPPS)Cl in a phosphate buffer solution at $\mathrm{pH} 7$ was checked by UV/vis spectrophotometry using a Perkin-Elmer Lambda 3B spectrophotometer in the range of 800 to 240 $\mathrm{nm}$. This solution showed an absorption maximum at 420 $\mathrm{nm}$ and a lower absorption at $390 \mathrm{~nm}$, in accordance with previous reports, ${ }^{27}$ and was stable at room temperature up to 10 months after preparation. The elemental analyses for $\mathrm{Fe}$ (TDCPPS)Cl $\left(\mathrm{Na}_{4} \mathrm{C}_{44} \mathrm{H}_{16} \mathrm{~N}_{4} \mathrm{O}_{12} \mathrm{~S}_{4} \mathrm{FeCl}_{9}\right)$ were as follows. Calculated (\%): Na, 6.63; C, 38.08; H, 1.15; N, 4.04; S, 9.24; Fe, 4.25. Measured (\%): Na, 6.59; C, 38.05; H, 1.18; N, 4.08; S, 9.27; Fe, 4.23.

Oxidative Polymerization of Humic Substances by Biomimetic Catalysis. Control humic solutions were prepared by re-dissolving $2.0 \mathrm{mg}$ of each sodium-humate sample in $8 \mathrm{~mL}$ of $0.1 \mathrm{M}$ phosphate buffer at $\mathrm{pH}$ 7. These solutions were treated with (1) $0.24 \mu \mathrm{mol}$ of Fe(TDCPPS)$\mathrm{Cl}$ catalyst $\left(2.2 \mathrm{~mL}\right.$ of a $1.09 \times 10^{-4} \mathrm{M}$ solution in Milli-Q water) to obtain a second control, (2) $0.41 \mathrm{mmol}$ of $\mathrm{H}_{2} \mathrm{O}_{2}$ (48 $\mu \mathrm{L}$ of a $8.6 \mathrm{M}$ freshly prepared solution) to obtain a third control, and (3) the same amounts of the Fe(TDCPPS)$\mathrm{Cl}$ catalyst and the $\mathrm{H}_{2} \mathrm{O}_{2}$ oxidizing agent to induce catalyzed oxidative coupling. For all solutions, the final volume was $10.248 \mathrm{~mL}$. The solutions were stirred and then incubated in darkness to prevent photooxidation. Preliminary highperformance size-exclusion chromatography (HPSEC) experiments indicated that oxidative polymerization did not increase after $100 \mathrm{~h}$ at room temperature. Hence, following $100 \mathrm{~h}$ of reaction time, the treated samples were filtered through a $0.2 \mu \mathrm{m}$ (PVDF Millipore) and injected into the HPSEC apparatus. The same control and polymerization solutions were then added with glacial acetic acid until pH 3.5 and injected again in the HPSEC systems. To conduct spectroscopic analyses, an adequate amount of sample (100 $\mathrm{mg}$ ) was subjected to the catalyzed oxidative polymerization for $100 \mathrm{~h}$. The mixture was first precipitated to $\mathrm{pH} 2$ by adding $6 \mathrm{M} \mathrm{HCl}$ and then centrifuged. The residue was dialyzed against Milli-Q water until $\mathrm{Cl}$-free and then freezedried.

Size-Exclusion Chromatography. The HPSEC system consisted of a high-pressure Perkin-Elmer LC200 solvent pump and of two detectors in series: a UV/vis variablewavelength detector (Perkin-Elmer LC295) set at $280 \mathrm{~nm}$ and a refractive index (RI) detector (Fisons Instruments, Refractomonitor IV). A rheodyne rotary injector, equipped with a $100 \mu \mathrm{L}$ sample loop, was used to load the calibration standard and humic solutions. Size-exclusion separation 
occurred through a Polysep GFC-P-3000 (600 mm per 7.5 $\mathrm{mm}$ i.d.) Phenomenex SEC column (Phenomenex, Inc., USA). The column was preceded by a $7.5 \mathrm{~cm}$ Phenomenex GFC-P Guard Column (7.5 mm i.d.) and by a $0.2 \mu \mathrm{m}$ stainless steel inlet filter. The column system was thermostated at $25^{\circ} \mathrm{C}$ in a water bath. The flow rate was set to 0.6 $\mathrm{mL} \cdot \mathrm{min}^{-1}$, and the HPSEC eluent was a solution of $0.1 \mathrm{M}$ $\mathrm{NaH}_{2} \mathrm{PO}_{4}$ buffered at $\mathrm{pH} 7$ with $0.5 \mathrm{M} \mathrm{NaOH}$ and added with $4.0 \times 10^{-3} \mathrm{M} \mathrm{NaN}_{3}$ as a bacteriostatic agent. The mobile phase was made with MilliQ water and HPLC-grade reagents, filtered through Millipore $0.45 \mu \mathrm{m}$, and $\mathrm{He}$ degassed. The void volume $\left(V_{0}=11.56 \mathrm{~mL}\right)$ and total permeation volume $\left(V_{\mathrm{t}}=24.80 \mathrm{~mL}\right)$ of the columns were determined using Blue Dextran 2000 and water, respectively.

Size-exclusion chromatograms for both the UV and RI detectors were evaluated by using Perkin-Elmer-Nelson Turbochrom 4-SEC peak integration and molecular weight software, a SEC noise threshold of 5, and a filter size of 5 for the Savitzky-Golay smoothing. Calculation of apparent weight-average $\left(\mathrm{Mw}_{\mathrm{a}}\right)$ molecular weights was done as previously described. ${ }^{3}$ The $\mathrm{Mw}_{\mathrm{a}}$ values from chromatograms of control and treated HAs as well as from chromatograms of the same HAs added with acetic acid were obtained by using calibration curves with standard polysaccharides (Polymer Sciences Laboratories, U.K.) of known molecular weights (100 000, 48 000, 23 700, and 12 200). The relative standard deviation of calculated values among triplicates of each chromatogram varied to a maximum of $7 \%$, thereby confirming the good reproducibility of the HPSEC system. ${ }^{3}$

CPMAS- ${ }^{13}$ C NMR Spectroscopy. Cross-polarization magic angle spinning carbon-13 nuclear magnetic resonance spectroscopy (CPMAS- ${ }^{13} \mathrm{C}$ NMR) experiments were carried out on a Bruker AMX300 spectrometer operating at 75.47 $\mathrm{MHz}$ on the carbon-13 and a rotor spin rate of $5000 \pm 10$ $\mathrm{Hz}$. A recycle time of $1 \mathrm{~s}$ and an acquisition time of $23 \mathrm{~ms}$ were used. All experiments were conducted with a variable contact time (VCT) pulse sequence, ${ }^{28}$ by allowing a contact time from 2 to $7 \mathrm{~ms}$ in sequential experiments from 2 to 4 h, acquiring 2000 scans for each spectrum, and applying a line broadening (LB) of $100 \mathrm{~Hz}$ to transform all the free induction decays (FID) in order to obtain a signal-to-noise $(S / N)$ ratio $\geq 20$. All spectra were integrated by the available software in the $0-230 \mathrm{ppm}$ range to evaluate total spectral areas $(I(t))$. The $I(t)$ values were used to calculate the total $T_{1 \rho}(\mathrm{H})$ values of the HS without and with the treatment with $\mathrm{Fe}$ (TDCPPS)Cl and $\mathrm{H}_{2} \mathrm{O}_{2}$ as follows:

$$
\ln I(t)=\ln I_{0}-t_{\mathrm{CP}} / T_{1 \rho}(\mathrm{H})
$$

where $t_{\mathrm{CP}}$ is the contact time, $I(t)$ is the signal area at the $t$-th contact time, and $T_{1 \rho}(\mathrm{H})$ is the proton spin-lattice relaxation time in the rotating frame. ${ }^{29}$ The total signal areas in these spectra were measured with the provided software, showing a relative standard deviation (RSD) of $<6 \%$.

Electron Paramagnetic Resonance. Electron paramagnetic resonance (EPR) spectra were recorded with a Bruker EMX Spectrometer, operating at X-band (9 GHz), with a $100 \mathrm{kHZ}$ magnetic field modulation frequency, microwave power of $0.2 \mathrm{~mW}$, at room temperature, and modulation amplitude of $0.5 \times 10^{-4} \mathrm{~T}$. The $g$ parameter was measured against 2,2-diphenyl-1-picryhydrazyl (DPPH) standard ( $g=$ 2.0036). For quantitative analysis EPR quartz tubes $(2.5 \mathrm{~mm}$ i.d.) were filled in duplicate with solid humic samples that were $0.5 \mathrm{~cm}$ high. To quantify the organic free radicals, a ruby crystal was used as a secondary standard, according to the Singer's method. ${ }^{30,31}$ Details for measurements of derivative peak-to-peak line width $(\Delta H)$ at microwave power saturations are reported elsewhere. ${ }^{32}$

DRIFT Spectroscopy. Diffuse reflectance infrared spectroscopy (DRIFT) was carried out on a Perkin-Elmer Spectrum-One FTIR spectrometer. All samples were prepared in the solid state by mixing $1 \mathrm{mg}$ of sample with 300 $\mathrm{mg}$ of $\mathrm{KBr}$ in an agate mortar. To acquire all spectra, 16 scans were used. Automatic subtraction of water, smoothing, and base line correction was achieved by the Perkin-Elmer Spectrum 5.0 FTIR software.

\section{Results and Discussion}

Sample Characteristics. The HAs of this study had different elemental and molecular characteristics, ${ }^{20}$ with the HA1 from an oxidized coal showing the highest degree of condensed or unsaturated carbon compounds $(\mathrm{C} / \mathrm{H}=16.0)$, followed by $\mathrm{HA} 2$ from a lignite $(\mathrm{C} / \mathrm{H}=12.4)$ and $\mathrm{HA} 3$ from a forested volcanic soil $(\mathrm{C} / \mathrm{H}=11.0)$. Confirming the measured $\mathrm{C} / \mathrm{H}$ values, CPMAS $-{ }^{13} \mathrm{C}$ NMR spectra ${ }^{20}$ indicated that HA3 had a higher content of polar carbons, such as oxidized (53-56 ppm) and carbohydrate (70-105 ppm) carbons, than HA1 and HA2. HA3 had also the highest content of alkyl carbons (30-32 ppm), whereas both HA1 and HA2 were significantly richer in aromatic and carboxyl carbons (around 130 and 170 ppm, respectively). Areas from different resonance intervals of NMR spectra ${ }^{20}$ were used to quantify potential humic reactivity due to polar (HI) and apolar carbons (HB). The HI/HB ratio varied in the following order: HA3 $>$ HA1 $>$ HA2, indicating that HA3 was the most potentially hydrophilic material, while HA2 was the most hydrophobic of the HAs. Aromaticity of these HS was also obtained from CPMAS- ${ }^{13} \mathrm{C}$ NMR spectra, ${ }^{20}$ resulting in the following order: HA2 $\geq$ HA1 $\gg$ HA3.

Size-Exclusion Chromatograms. It is assumed that the hydrophobic humic components in aqueous solutions should be pulled together and isolated from water in order to decrease the total free energy of solution, ${ }^{33,34}$ whereas the hydrophilic constituents should preferentially accommodate in the outer sites of humic associations. The heterogeneity of humic molecules determines a particular supramolecular conformation in water solution depending on the affinity among specific molecular structures and their associated hydration water. The particular conformational arrangement of a humic association in solution is then reflected in a molecular size distribution that is summarized in $\mathrm{Mw}_{\mathrm{a}}$ as measured by HPSEC. ${ }^{3}$ Values of $\mathrm{Mw}_{\mathrm{a}}$ show that HA3 had the largest apparent molecular dimension followed by HA2 and HA1 (Table 1). These differences may be attributed to the large $\mathrm{HI} / \mathrm{HB}$ ratio of HA3. The hydrophilicity of HA3 favors the most hydrated conformation among the humic materials and, hence, structural associations in solution with the largest dimension and thus largest $\mathrm{Mw}_{\mathrm{a}}$. By contrast, HA1 
Table 1. Apparent Weight-Average Molecular Weight $\left(\mathrm{Mw}_{\mathrm{a}}\right)$ Values and Their Percent Variation $(\Delta)$ of Humic Samples: ${ }^{a}$ Control, Control + $\mathrm{Fe}$ (TDCPPS) $\mathrm{Cl}$ (Control + FePo), Control $+\mathrm{H}_{2} \mathrm{O}_{2}$, Control $+\mathrm{FePo}+\mathrm{H}_{2} \mathrm{O}_{2}$ (Polymer), without (pH 7) and with (pH 3.5) Addition of Acetic Acid $(\mathrm{AcOH})$

\begin{tabular}{|c|c|c|c|c|c|c|c|c|}
\hline & control & $\begin{array}{c}\text { control }+ \\
\mathrm{AcOH}\end{array}$ & $\begin{array}{c}\text { control }+ \\
\text { FePo }\end{array}$ & $\begin{array}{c}\text { control }+ \\
\text { FePo }+\mathrm{AcOH} \\
\end{array}$ & $\begin{array}{c}\text { control }+ \\
\mathrm{H}_{2} \mathrm{O}_{2} \\
\end{array}$ & $\begin{array}{c}\text { control }+ \\
\mathrm{H}_{2} \mathrm{O}_{2}+\mathrm{Ac} \mathrm{OH} \\
\end{array}$ & polymer & $\begin{array}{c}\text { polymer }+ \\
\mathrm{AcOH} \\
\end{array}$ \\
\hline \multicolumn{9}{|c|}{ HA1 } \\
\hline $\begin{array}{l}\mathrm{Mwa}_{\mathrm{a}} \\
\Delta\end{array}$ & $1449 \pm 63$ & $\begin{array}{l}265 \pm 16 \\
-81.7^{b}\end{array}$ & $1427 \pm 42$ & $\begin{array}{l}261 \pm 24 \\
-81.7^{b}\end{array}$ & $1478 \pm 36$ & $\begin{array}{l}266 \pm 9 \\
-82.0^{b}\end{array}$ & $\begin{array}{l}4734 \pm 181 \\
+226.7^{b}\end{array}$ & $\begin{array}{l}1558 \pm 89 \\
+7.5,^{b}+487.9^{c}\end{array}$ \\
\hline \multicolumn{9}{|c|}{$\mathrm{HA} 2$} \\
\hline $\begin{array}{l}\mathrm{Mwa}_{\mathrm{a}} \\
\Delta\end{array}$ & $1469 \pm 58$ & $\begin{array}{l}269 \pm 15 \\
-81.7^{b}\end{array}$ & $1476 \pm 33$ & $\begin{array}{l}273 \pm 22 \\
-81.5^{b}\end{array}$ & $1451 \pm 24$ & $\begin{array}{l}270 \pm 13 \\
-81.3^{b}\end{array}$ & $\begin{array}{l}4112 \pm 219 \\
+179.9^{b}\end{array}$ & $\begin{array}{l}1641 \pm 106 \\
+11.7, b+510.0^{c}\end{array}$ \\
\hline \multicolumn{9}{|c|}{ HA3 } \\
\hline $\begin{array}{l}\mathrm{Mw}_{\mathrm{a}} \\
\Delta\end{array}$ & $4119 \pm 188$ & $\begin{array}{l}2151 \pm 113 \\
-47.7^{b}\end{array}$ & $4177 \pm 59$ & $\begin{array}{l}2194 \pm 38 \\
-47.5^{b}\end{array}$ & $4156 \pm 31$ & $\begin{array}{l}2178 \pm 66 \\
-47.5^{b}\end{array}$ & $\begin{array}{l}8399 \pm 371 \\
+103.9^{b}\end{array}$ & $\begin{array}{l}3456 \pm 255 \\
-16.1, b+60.6^{c}\end{array}$ \\
\hline
\end{tabular}

${ }^{a}$ HA1, humic acid from an oxidized coal; HA2, humic acid from a lignite; HA3, humic acid from a volcanic soil. ${ }^{b}$ Percent variation with respect to the control solution (control). ${ }^{c}$ Percent variation with respect to the acid-treated control solution (control $\left.+\mathrm{AcOH}\right)$.

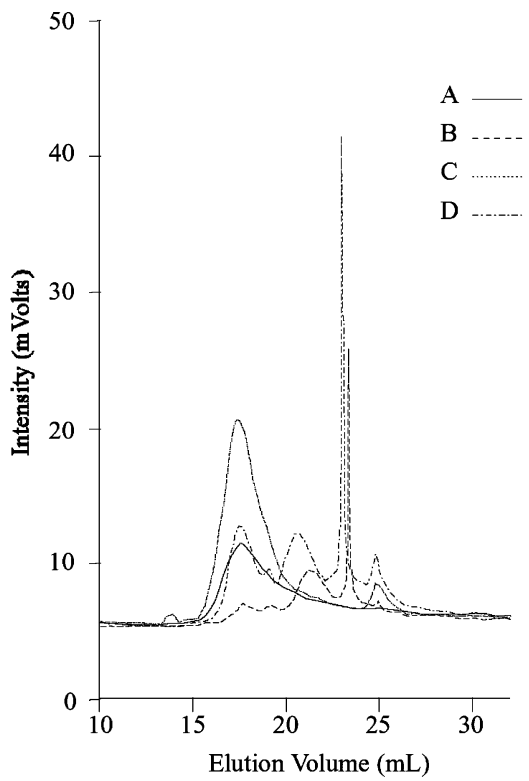

Figure 1. HPSEC chromatograms of the control HA1 solution (HA from an oxidized coal) in phosphate buffer at $\mathrm{pH} 7(\mathrm{~A})$, of the same solution as in $\mathrm{A}$ but brought to $\mathrm{pH} 3.5$ with acetic acid (B), of the same solution as in $\mathrm{A}$ but added with both $\mathrm{Fe}$ (TDCPPS) $\mathrm{Cl}$ and $\mathrm{H}_{2} \mathrm{O}_{2}$ (C), and of the same solution as in $\mathrm{C}$ but brought to $\mathrm{pH} 3.5$ with acetic acid (D).

and HA2, which reveal similar $\mathrm{Mw}_{\mathrm{a}}$ values, have smaller $\mathrm{HI} / \mathrm{HB}$ ratios, thereby indicating a more hydrophobic nature than HA3. This implies less hydrated conformations in water solution, more compact associations, and smaller $\mathrm{Mw}_{\mathrm{a}}$ values for HA1 and HA2. ${ }^{20}$

Figures $1-3$ show the size-exclusion chromatograms of humic solutions without and with the oxidative polymerization catalyzed by $\mathrm{Fe}$ (TDCPPS)Cl. The chromatograms of the humic solutions added just with either $\mathrm{Fe}$ (TDCPPS)Cl or $\mathrm{H}_{2} \mathrm{O}_{2}$ did not differ significantly (not shown) from those of control solutions. The control chromatograms were modified by lowering the $\mathrm{pH}$ of the humic solution from 7 to 3.5 with acetic acid prior to the injection in the HPSEC system. A similar amount of acetic acid $\left(<10^{-6} \mathrm{M}\right)$ did not affect the HPSEC elution profiles of macropolymers such as polysaccharides and polystirenesulfonates. ${ }^{35,36}$ For both HA1 and HA2 (Figures 1 and 2), the diffuse peak centered at about $18 \mathrm{~mL}$ of elution volume in control chromatograms was dramatically reduced, while the appearance of three new

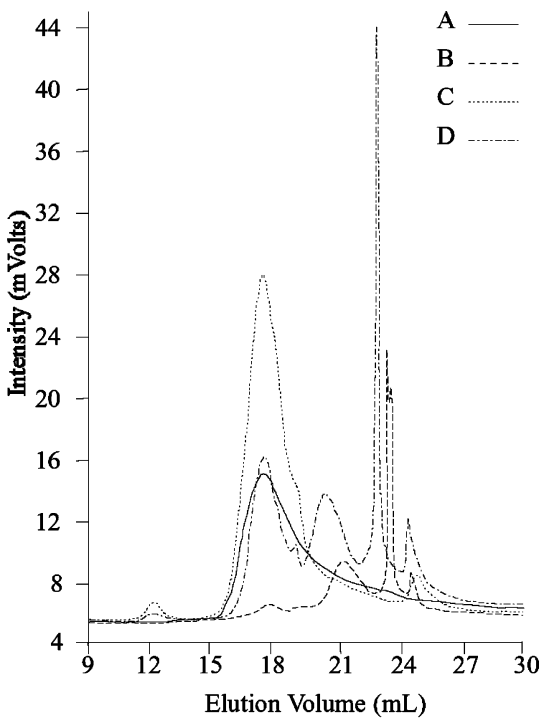

Figure 2. HPSEC chromatograms of the control HA2 solution (HA from a lignite) in phosphate buffer at $\mathrm{pH} 7(\mathrm{~A})$, of the same solution as in $\mathrm{A}$ but brought to $\mathrm{pH} 3.5$ with acetic acid (B), of the same solution as in $\mathrm{A}$ but added with both $\mathrm{Fe}(\mathrm{TDCPPS}) \mathrm{Cl}$ and $\mathrm{H}_{2} \mathrm{O}_{2}(\mathrm{C})$, and of the same solution as in $\mathrm{C}$ but brought to $\mathrm{pH} 3.5$ with acetic acid (D).

chromatographic peaks was noticeable at higher elution volumes. The second of these new peaks, at about $23.5 \mathrm{~mL}$, was particularly sharp and of higher intensity than the main diffuse peak visible in the control chromatograms. For HA3 (Figure 3), from a volcanic soil, the addition of acetic acid modified the chromatograms to a lesser extent. The main diffuse chromatographic peak was only slightly reduced, though shifted to larger elution volumes, and the new three peaks, also visible here, appeared less intense than for HA1 and HA2. The occurrence of the sharp and intense peak at around $23.5 \mathrm{~mL}$ of elution volume was not observed for the same humic materials when they had been analyzed by HPSEC with a TSK G3000SW column in similar conditions,$^{20}$ thereby suggesting a particular resolution of the Phenomenex column used here.

The HPSEC behavior of HAs following the treatment with acetic acid cannot be attributed to a modification of the column separation capacity because of the low amount of the acid added to the injected sample. ${ }^{36}$ It is, instead, attributed to a conformational change of the humic supramolecular structure previously stabilized at $\mathrm{pH} 7$ by weak 


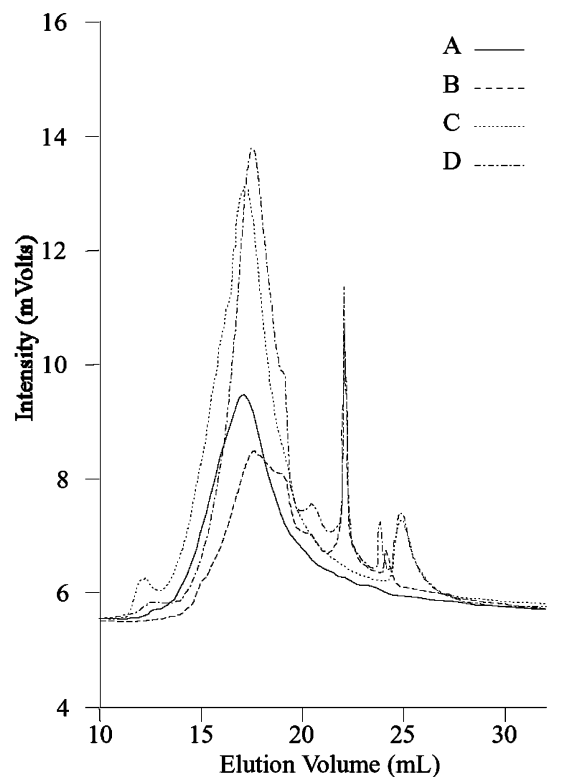

Figure 3. HPSEC chromatograms of the control HA3 solution (HA from a volcanic soil) in phosphate buffer at $\mathrm{pH} 7(\mathrm{~A})$, of the same solution as in $\mathrm{A}$ but brought to $\mathrm{pH} 3.5$ with acetic acid (B), of the same solution as in A but added with both $\mathrm{Fe}$ (TDCPPS) Cl and $\mathrm{H}_{2} \mathrm{O}_{2}$ (C), and of the same solution as in $\mathrm{C}$ but brought to $\mathrm{pH} 3.5$ with acetic acid (D).

bondings. Due to formation of intra- and intermolecular hydrogen bonds upon $\mathrm{pH}$ decrease, the stability of the original humic association was modified and eluted differently through the HPSEC column. ${ }^{3}$ It is plausible that some specific humic molecules or their smaller size associations, which were previously loosely stabilized into an apparently larger conformation at $\mathrm{pH} 7$, were liberated by the interaction with acetic acid and appeared as distinct peaks at larger elution volumes. In fact, the sharp and intense appearance of a peak eluted at about $23.5 \mathrm{~mL}$ for all humic materials may well be attributed to a homogeneous class of humic components. The modification of the HPSEC chromatograms induced by acetic acid addition was reflected by the $\mathrm{Mw}_{\mathrm{a}}$ values (Tab. 1). Both control and control $+\mathrm{Fe}(\mathrm{TDCPPS}) \mathrm{Cl}$ solutions of HA1 and HA2 showed a reduction of the apparent weight-average molecular weight of about $82 \%$ after acetic acid treatment, whereas that of the same solutions of HA3 was about $48 \%$. These results confirm that the addition of organic acids to a humic solution in combination with HPSEC analysis is useful in investigating the specific conformational stability of humic associations.

After the humic samples had undergone oxidative polymerization catalyzed by $\mathrm{Fe}$ (TDCPPS)Cl, the shape of the HPSEC chromatograms was further modified. For all three HAs (Figures 1-3), the diffuse chromatographic peak similar to that of control chromatograms resulted in enhanced intensity and shifted elution to lower volumes. Moreover, the appearance of one peak at about void volume and another one at around the total volume of the HPSEC column was observed. These changes mainly suggest an increase in the dimension of the molecular size distribution of all humic samples following the oxidative polymerization reaction. Larger molecular sizes were indicated by the $\mathrm{Mw}_{\mathrm{a}}$ values (Table 1), which were enhanced by 227,180 , and $104 \%$ in comparison to those of control chromatograms for HA1, HA2, and HA3, respectively.

When the solution $\mathrm{pH}$ was lowered to 3.5 with acetic acid, the HPSEC chromatograms (Figures 1-3) were further modified in comparison to those of the unpolymerized samples. For HA1 and HA2, the acetic acid addition was unable to reduce the diffuse chromatographic peak, at about $18 \mathrm{~mL}$ of elution volume, to the same extent as in the acidtreated samples without the catalyzed oxidation treatment. Moreover, this peak was still more intense than the control solutions. For HA3, a similar diffuse peak was more intense than those of both the acid-treated and -untreated control solutions, while a noticeable peak remained visible at the void volume for the acid-treated polymerized sample (Figure 3D). As in the case of the acid-treated control samples, three further peaks at larger elution volumes, including an intense and sharp one, were produced by the action of acetic acid on the polymerized humic samples. However, for HA1 and HA2, these peaks were even somewhat shifted to lower elution volumes than for control samples. The observed chromatographic modifications were reflected in the calculated $\mathrm{Mw}_{\mathrm{a}}$ values which showed a significant increase with respect to those found for both control and control $+\mathrm{Fe}-$ (TDCPPS)Cl samples (Table 1). In particular, the $\mathrm{Mw}_{\mathrm{a}}$ values were 488,510 , and $61 \%$ larger than the acid-treated unpolymerized samples for HA1, HA2, and HA3, respectively.

These results suggest that, due to the catalyzed oxidative reaction, the humic supramolecular associations acquired a significantly higher stability in comparison with control materials. As observed for the catalytic effect of peroxidase, ${ }^{17,18}$ the oxidative catalysis by the biomimetic Fe(TDCPPS)Cl may have stabilized the humic associations through the formation of new covalent $\mathrm{C}-\mathrm{O}-\mathrm{C}$ or $\mathrm{C}-\mathrm{C}$ bonds. The fact that the conformations of the samples subjected to the oxidative reaction were not modified by the acetic acid treatment as extensively as for both control samples may well be evidence for the occurred oxidative coupling via covalent bonds among humic molecules. The different behavior of the three humic samples should be accounted to their diverse molecular composition and hydration size in solution. ${ }^{3,20}$

NMR Spectra and Proton Spin-Lattice Relaxation Time. Solid-state nuclear magnetic resonance (CPMAS- ${ }^{13} \mathrm{C}$ NMR) spectra of the HA1 and HA3 samples with and without the polymerization treatment are shown in Figure 4. The polymerization reaction appears to have reduced carboxylic carbons (173 ppm) and slightly enhanced alkyl carbons $(30 \mathrm{ppm})$ in HA1, whereas a small increase of phenolic carbons (151 ppm) and a decrease of aromatic (130 $\mathrm{ppm})$ and alkyl (33 ppm) carbons was noticed for HA3. The HA2 sample (not shown) behaved similarly to the HA1 sample. The changes observed in these NMR spectra were not informative, as expected, on the molecular variations induced by the polymerization reaction. This should be attributed to the poor resolution of solid-state NMR spectra of complex humic molecules.

However, CPMAS- ${ }^{13} \mathrm{C}$ NMR spectroscopy may be used to characterize the dynamics of solids at a molecular level, 


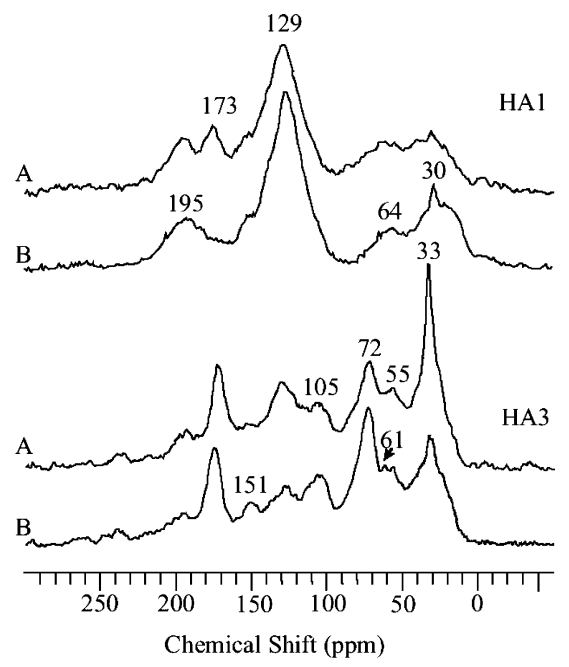

Figure 4. CPMAS- ${ }^{13} \mathrm{C}$ NMR spectra of $\mathrm{HA} 1$ and HA3 samples without $(A)$ and with (B) the oxidative polymerization by $\mathrm{Fe}$ (TDCPPS)$\mathrm{Cl}$ biomimetic catalyst.

being very sensitive to the nanoscale morphology of molecular segments. ${ }^{29,37}$ In particular, the proton spin-lattice relaxation time in the rotating frame, $T_{1 \rho}(\mathrm{H})$, spatially averaged over several tens of nanometers due to spin diffusion, is dependent on molecular morphology. The $T_{1 \rho^{-}}$ $(\mathrm{H})$ value measured for a sample should be a molar-averaged value of the $T_{1 \rho}(\mathrm{H})$ component of the amorphous fraction and the $T_{1 \rho}(\mathrm{H})$ component of a less amorphous, semicrystalline, fraction. ${ }^{38}$ When $T_{1 \rho}(\mathrm{H})$ is measured by the variable contact-time experiment (VCT), this parameter is obtained from the intensity decay for all resolved carbons. Thus, one obtains the value of $T_{1 \rho}(\mathrm{H})$ for all types of hydrogen, which permits evaluation of the overall mobility behavior of the sample. The $T_{1 \rho}(\mathrm{H})$ values measured from the VCT are intimately linked with the process of polarization transfer and to the chemical shift values for the type of carbons that are linked. As the $T_{1 \rho}(\mathrm{H})$ values are also sensitive to the proximity of protonated segments to one another, the distance between two protonated segments are directly related to the proton-proton spin diffusion. ${ }^{39,40}$ It is thus conceivable that a change in the mutual spatial relations of humic protonated segments passing from a loosely bound supramolecular association into a more rigid structure brought about by a catalyzed oxidative polymerization may be shown by a difference in $T_{1 \rho}(\mathrm{H})$ values.

The $T_{1 \rho}(\mathrm{H})$ values obtained by VCT experiments for humic samples with and without the oxidative treatment catalyzed by $\mathrm{Fe}(\mathrm{TDCPPS}) \mathrm{Cl}$ are shown in Table 2 . The $T_{1 \rho}(\mathrm{H})$ values for the control samples were 6.0, 7.9, and $5.7 \mathrm{~ms}$ for HA1,
HA2, and HA3, respectively. The oxidative catalyzed reaction produced samples showing significantly larger $T_{1 \rho}(\mathrm{H})$ values up to $9.9,28.5$, and $20.1 \mathrm{~ms}$ for the HA1, HA2, and HA3 samples, respectively. This increase in $T_{1 \rho}(\mathrm{H})$ values after the oxidative reaction cannot be attributed to the paramagnetic effect arising from the little amount of $\mathrm{Fe}$ introduced with $\mathrm{Fe}(\mathrm{TDCPPS}) \mathrm{Cl}$. The $T_{1 \rho}(\mathrm{H})$ value was found to be rather insensitive to paramagnetic species in humic samples ${ }^{41}$ and also it should decrease more than increase upon a significant effect of paramagnetic iron. ${ }^{42}$ The enhancement of $T_{1 \rho}(\mathrm{H})$ values is rather due to the increased motional rigidity of humic molecules caused by the formation of covalent intermolecular linkages and consequent larger molecular mass than for control. Similarly, the increase of $T_{1 \rho}(\mathrm{H})$ values in humic matter amended with divalent cations was attributed to the reduced molecular mobility brought about by the intermolecular chelation of cations. ${ }^{41}$ As recently shown, free divalent metals are responsible for intermolecular complex linkages among low molecular-weight humic molecules. ${ }^{9}$ The increase in $T_{1 \rho}(\mathrm{H})$ values found here for the humic samples subjected to the oxidative catalyzed reaction agree with the HPSEC results in suggesting that the Fe(TDCPPS)Cl catalysis was able to covalently polymerize humic molecules and promote more rigid humic conformations than in the weakly bound supramolecular structures of control samples.

Electron Paramagnetic Resonance Spectra. The main parameters obtained from EPR spectra are shown in Table 2. The $g$ values, attributed to organic free radicals,${ }^{31}$ did not increase with addition of $\mathrm{Fe}$ (TDCPPS)Cl, indicating an insignificant effect of paramagnetic $\mathrm{Fe}^{3+}$ ions. The microwave power at half-saturation $\left(P_{1 / 2}\right)$ showed a significant decrease passing from control to humic samples subjected to the oxidative catalyzed reaction, while the line width $(\Delta H)$ values varied only slightly. The $\Delta H$ values are currently related to $T_{1}$ (spin-lattice) and $T_{2}$ (spin-spin) relaxation times through an inverse relationship of the microwave power at $P_{1 / 2}$ with $T_{1}$ and $T_{2}{ }^{43}$ It was suggested that, for humic substances, $\Delta H$ is more sensitive to $T_{2}$ variation, whereas changes in $P_{1 / 2}$ reflect variations in $T_{1}{ }^{32,43}$ Our results, a slight modification in $\Delta H$ and a large decrease in $P_{1 / 2}$, suggest that a significant change should have occurred with polymerization only for $T_{1}$, but not for $T_{2}$, thereby indicating a sensitivity of $T_{1}$ to the increase in molecular mass for the polymerized samples. This behavior of relaxation times can be related to a reduced mobility of humic molecules subjected to the catalyzed oxidative reaction, ${ }^{44}$ thereby further substantiating the hypothesis that the $\mathrm{Fe}$ (TDCPPS)Cl catalysis

Table 2. EPR Values for $g$ Parameter, Line Width $(\Delta H)$, Spin Concentration, and Microwave Power at Half-Saturation $\left(P_{1 / 2}\right)$ and CPMAS- ${ }^{13} \mathrm{C}$ NMR Values for Proton Spin-Lattice Relaxation Time in the Rotating Frame, $T_{1 \rho}(\mathrm{H})$, of Humic Samples without (Control) and with Polymerization Treatment

\begin{tabular}{clcccrr}
\hline sample $^{a}$ & treatment & $\mathrm{g}$ & $\Delta H(\mathrm{mT})$ & $\mathrm{spin} \mathrm{g}^{-1}\left(10^{17}\right)$ & $P_{1 / 2(\mathrm{max})}$ & $T_{1 \rho}(\mathrm{H})(\mathrm{ms})$ \\
\hline $\mathrm{HA} 1$ & control & 2.0038 & $0.56 \pm 0.007$ & $4.22 \pm 0.182$ & 7.01 & $6.0 \pm 0.4$ \\
& polymerized & 2.0036 & $0.62 \pm 0.003$ & $4.32 \pm 0.106$ & 1.47 & $9.9 \pm 0.6$ \\
$\mathrm{HA2}$ & control & 2.0036 & $0.53 \pm 0.000$ & $1.29 \pm 0.0625$ & 8.16 & $7.9 \pm 0.5$ \\
& polymerized & 2.0036 & $0.55 \pm 0.000$ & $2.15 \pm 0.0453$ & 1.43 & $28.5 \pm 1.7$ \\
$\mathrm{HA3}$ & control & 2.0036 & $0.49 \pm 0.004$ & $1.90 \pm 0.0185$ & 6.50 & $5.7 \pm 0.3$ \\
& polymerized & 2.0036 & $0.53 \pm 0.008$ & $2.72 \pm 0.0565$ & 1.49 & $20.1 \pm 1.2$ \\
\hline
\end{tabular}

${ }^{a}$ HA1, humic acid from an oxidized coal; HA2, humic acid from a lignite; HA3, humic acid from a volcanic soil. 
A

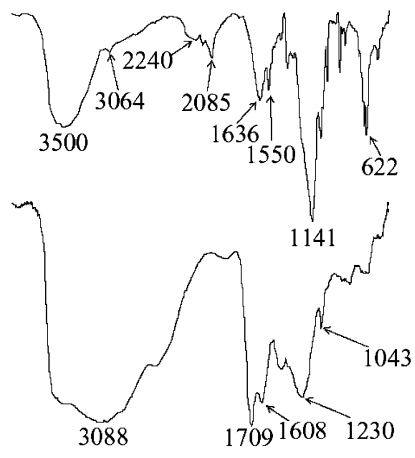

C

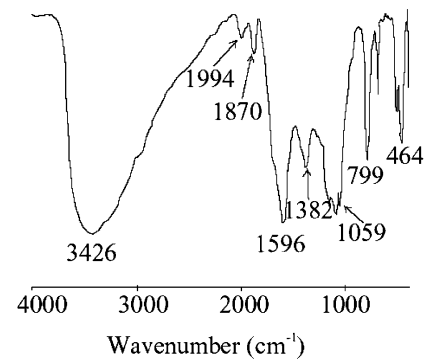

Figure 5. DRIFT spectra of $\mathrm{Fe}(\mathrm{TDCPPS}) \mathrm{Cl}$ alone (A), control HA1 (B), and polymerized HA1 (C) samples.

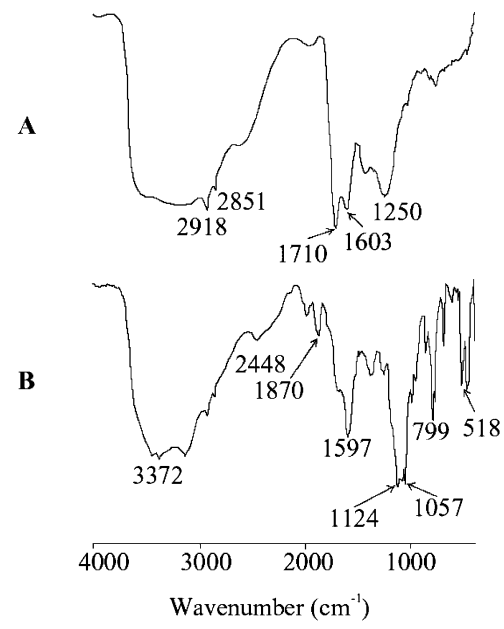

Figure 6. DRIFT spectra of control HA2 (A) and polymerized HA2 (B) samples.

increased the humic molecular mass by an intermolecular oxidative polymerization. This proposal is also consistent with the increase of organic free-radical content observed in the polymerized samples.

DRIFT Spectra. DRIFT (diffuse reflectance infrared Fourier transform) spectra of fine mixtures with $\mathrm{KBr}$ of $\mathrm{Fe}$ (TDCPPS)Cl alone, control HA1, and oxidative-treated HA1 are shown in Figure 5, while those of control HA2 with treated HA2, and control HA3 with treated HA3, are reported in Figures 6 and 7, respectively. Complete bands attribution for DRIFT spectra of humic materials are reported elsewhere ${ }^{45}$ whereas absorptions for $\mathrm{Fe}$ (TDCPPS)Cl (Figure 5) at $3064 \mathrm{~cm}^{-1}$ and in the regions of 1500-2200 and 1000$1200 \mathrm{~cm}^{-1}$ can be assigned to benzene rings, pyrroles, and benzenesulfonic acids, respectively. ${ }^{46,47}$

In comparison with control HA1 and HA2, the DRIFT spectra of the same material subjected to oxidative catalysis with $\mathrm{Fe}(\mathrm{TDCPPS}) \mathrm{Cl}$ showed a substantial change in the $900-1500 \mathrm{~cm}^{-1}$ frequency interval (Figures 5 and 6). The broad absorption at about $1230-1250 \mathrm{~cm}^{-1}$, attributable
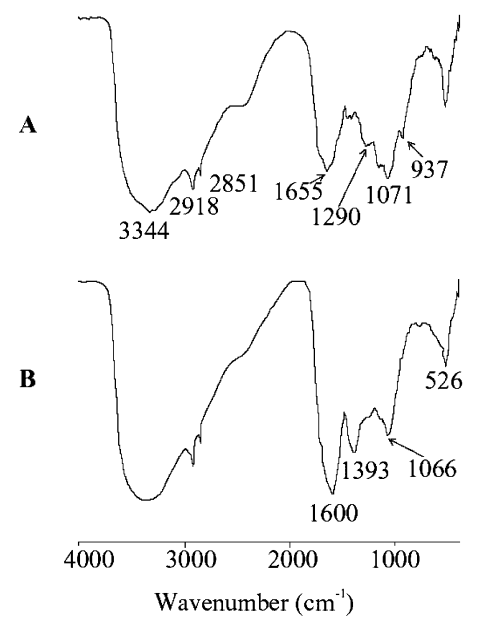

Figure 7. DRIFT spectra of control HA3 (A) and polymerized HA3 (B) samples.

mainly to phenols $\mathrm{CO}$ deformation, was changed into another broad band with distinct peaks from 1158 to $1059 \mathrm{~cm}^{-1}$ which can be reasonably assigned to $\mathrm{C}-\mathrm{O}-\mathrm{C}$ bond deformation of alkyl and aryl ethers. ${ }^{46,47}$ Moreover, the bands at $1870 \mathrm{~cm}^{-1}$ in the catalyst-treated samples of HA1 and HA2 may be assigned to cyclic anhydrides formed following oxidative cleavages of aromatic rings promoted by the biomimetic catalyst. ${ }^{46}$ For HA3, the variation from control to the oxidative-coupled sample was less striking (Figure 7), although the $1290 \mathrm{~cm}^{-1}$ band of this material, visible in the control spectrum, was substantially modified and an absorption centered at $1066 \mathrm{~cm}^{-1}$, also possibly assignable to aromatic ethers, was noticed in the spectrum of the reacted sample. The difference in DRIFT spectra between HA3 and the other two samples may be also ascribed to their diversity in aromaticity and, thus, phenol content assessed by CPMAS${ }^{13} \mathrm{C}$ NMR spectroscopy. However, the information acquired by DRIFT spectroscopy on the formation of alkyl and aryl ethers in polymerized humic samples appears in agreement with the evidence of increased molecular dimension and rigidity acquired by other chromatographic and spectroscopic results.

Our results indicate that a water-soluble iron-porphyrin catalyzes the oxidative transformation of weakly bound humic superstructures into stable conformations of larger molecular size through formation of covalent bonds among the reactive humic molecules. This effect was not significantly observed when the biomimetic catalyst was not combined with an oxidizing agent. The HPSEC elution profiles showed that the supramolecular conformations, which were easily separated into smaller components by addition of acetic acid to control samples, were no longer disrupted by the acetic acid action after the oxidative coupling catalyzed by iron-porphyrin. The catalytic activity of iron-porphyrin in the oxidative coupling of humic components appears to produce a larger effect on the humic size distribution than that of peroxidase. ${ }^{20}$ DRIFT spectra suggested the formation of new covalent bonds of humic molecules such as aryl and alkyl ethers as a result of oxidative catalysis by iron-porphyrin. The occurrence of intermolecular polymerization in humic material was also implied by the increased molecular rigidity with respect to 
control shown by CPMAS- ${ }^{13} \mathrm{C}$ NMR and ESR parameters. These findings point out that the use of synthetic ecologically compatible biomimetic catalyzers such as metal-porphyrins may become important to regulate the chemical and biological properties of natural organic matter.

Acknowledgment. This work was partially supported by the Italian Ministry of University and Scientific and Technological Research (MURST). The EPR measurements by Dr. L. Martin-Neto of Embrapa, Instrumentação Agropecuária, São Carlos, Brazil, are gratefully acknowledged.

\section{References and Notes}

(1) Galeska, I.; Hickey, T.; Moussy, F.; Kreutzer, D.; Papadimitrakopoulos, F.; Biomacromolecules 2001, 2, 1249-1255.

(2) Fredheim, G. E.; Christensen, B. E. Biomacromolecules 2003, 4, 232-239.

(3) Conte, P.; Piccolo, A. Environ. Sci. Technol. 1999, 33, 1682-1690.

(4) Piccolo, A. Adv. Agron. 2002, 75, 57-134.

(5) Simpson, A. J.; Kingery, W. L.; Shaw, D. R.; Spraul, M.; Humpfer, E.; Dvortsak, P. Environ. Sci. Technol. 2001, 35, 3321-3325.

(6) Simpson, A. J. Magn. Reson. Chem. 2002, 40, S72-S82

(7) Piccolo, A.; Conte, P.; Cozzolino, A. Eur. J. Soil Sci. 1999, 50, 687694.

(8) Cozzolino, A.; Conte, P.; Piccolo, A. Soil Biol. Biochem. 2001, 33, $563-571$

(9) Wrobel, K.; Sadi, B. B. M.; Wrobel, K.; Castello, J. R.; Caruso, J. A. Anal. Chem. 2003, 75, 761-767.

(10) Kenworthy, I. P.; Hayes, M. H. B. In Humic Substances, Peats and Sludges; Hayes, M. H. B., Wilson, W. S., Eds.; The Royal Society of Chemistry: Cambridge, U.K., 1997; pp 39-45.

(11) Buurman, P.; van Lagen, B.; Piccolo, A. Org. Geochem. 2002, 33, 367-381

(12) Piccolo, A.; Conte, P.; Trivellone, E.; van Lagen, B.; Buurman, P. Environ. Sci. Technol. 2002, 36, 76-84.

(13) Piccolo, A.; Spiteller, M. Anal. Biochem. 2003, 377, 1047-1059.

(14) Kurioka, H.; Komatsu, I.; Uyama, H.; Kobayashi, S. Macromol. Rapid Commun. 1994, 15, 507-510.

(15) Wang, P.; Martin, B. D.; Parida, S.; Rethwisch, D. G.; Dordick, J. S. J. Am. Chem. Soc. 1995, 117, 12885-12886.

(16) Wang, P.; Dordick, J. S. Macromolecules 1998, 31, 941-943.

(17) Oguchi, T.; Tawaki, H.; Uyama, S.-i.; Kobayashi, S. Macromol. Rapid Commun. 1999, 20, 401-403.

(18) Peralta-Zamora, P.; Pereira, C. M.; Tiburtius, E. R. L.; Moraes, S. G.; Rosa, M. A.; Minussi, R. C.; Durań, N. Appl. Catal. B: Environm. 2003, 42, 131-144.

(19) Piccolo, A.; Cozzolino, A.; Conte, P.; Spaccini, R. Naturwissenschaften 2000, 87, 391-394.

(20) Cozzolino, A.; Piccolo, A. Org. Geochem. 2002, 33, 281-294.

(21) Labat, G.; Seris, J.-L.; Meunier, B. Angew. Chem., Int. Ed. Engl. 1990, 29, 1471-1473.
(22) Cozzolino, A.; Piccolo, A.; Ditmmar, H.; Tagliatesta; P.; Armenante, P. Advances in Environmental Technology, Workshop of the Annual Meeting of the American Institute of Chemical Engineers (AIChE), Indianapolis, IN., Nov. 3-8, 2002.

(23) Fukushima, M.; Sawada, A.; Kawasaki, M.; Uichikawa, H.; Morimoto, K.; Tatsumi, K.; Aoyama, M. Environ. Sci. Technol. 2003, 37, 1031-1036.

(24) Traylor, P. S.; Dolphin, D.; Traylor T. G. J. Chem. Soc., Chem. Commun. 1984, 279-280.

(25) Song, R.; Sorokin, A.; Bernardou, J.; Meunier, B. J. Org. Chem. 1997, 62, 673-678.

(26) Sheldon, R. A. Metalloporphyrins in Catalytic Oxidations; Dekker: New York, 1994; pp 12-13.

(27) Meunier, B.; Sorokin, A. Acc. Chem. Res. 1997, 30, 470-476.

(28) Conte, P.; Piccolo, A.; van Lagen, B.; Buurman, P.; Hemminga, M. A. Solid State Nucl. Magn. Reson. 2002, 21, 158-170.

(29) Stejskal, E. O.; Memory, J. D. High-Resolution NMR in the Solid State; Oxford University Press: New York, 1994; pp 1-186.

(30) Singer, L. S. J. Appl. Phys. 1959, 30, 1463-1464.

(31) Martin-Neto, L.; Nascimento, O. R.; Telamoni, J.; Poppi, N. R. Soil Sci. 1991, 51, 369-376.

(32) Novotny, E. H.; Martin-Neto, L. Geoderma 2002, 106, 305-317.

(33) Tanford, C. The Hydrophobic Effect: Formation of Micelles and Biological Membranes; Krieger: Malabar, FL, 1991.

(34) Schwarzenbach, R. P.; Gschwend, P. M.; Imboden, D. M. Environmental Organic Chemistry; Wiley: NewYork, 1993.

(35) Piccolo, A.; Conte, P.; Cozzolino, A. In Humic Substances. Versatile Components of Plant, Soils and Water; Gabbhour, E. A., Davies, G., Eds.; Special Publication No. 259; Royal Society of Chemistry: Cambridge, U.K., 2000; pp 111-124.

(36) Piccolo, A.; Conte, P.; Cozzolino, A. Soil Sci. 2001, 166, 174-185.

(37) Schaefer, J.; Stejskal, E. O.; Buchdal, R. Macromolecules 1975, 8, 291-296.

(38) McBrierty, V. J. In Comprehensive Polymer Science; Allen, G., Ed.; Pergamon Press: Oxford, U.K., 1989; Vol. 1, pp 397-428.

(39) Zumbulyadis, N. J. Magn. Reson. (1969-1992) 1983, 53, 486-494.

(40) Newman, R. H.; Condron, L. M. Solid State Nucl. Magn. Reson. 1995, 14, 1-8.

(41) Smernik, R. J.; Oades, J. M. J. Environ. Qual. 2002, 31, 414-420.

(42) Wilson, M. A. NMR Techniques and Applications in Geochemistry and Soil Chemistry, 1st ed.; Pergamon Press: London, 1987.

(43) Galli, C.; Innes, J. B.; Hirsch, D. J.; Brudvig, G. W. J. Magn. Res., Ser. B 1996, 110, 284-287.

(44) Poole, C. P., Jr. Electron Spin Resonance: A Comprehensive Treatise on Experimental Techniques, 2nd ed.; Wiley: New York, 1983.

(45) Spaccini, R.; Zena Teshale, A.; Piccolo, A. Fresenius Environ. Bull. 1998, 7, 458-465

(46) Bellamy, L. J. The Infrared Spectra of Complex Molecules, 3rd ed.; Wiley: New York, 1975; Vol. 1.

(47) Smith, L. A. Applied Infrared Spectroscopy. Fundamentals, Techniques and Analytical Problem-Solving; Wiley: New York, 1979.

BM0495203 